\title{
MODELAGEM E SIMULAÇÃO DE UM SISTEMA DE EVAPORAÇÃO MÚLTIPLO EFEITO
}

\author{
M. C. A. OLIVEIRA ${ }^{1}$, L. A. F. OLIVEIRA ${ }^{1}$, M. O. $\operatorname{AGUIAR}^{2}$, C. R. S. N. ALMEIDA ${ }^{3}$, G. \\ M. ALMEIDA ${ }^{2}$ \\ ${ }^{1}$ Universidade Federal de São João del-Rei, Departamento de Engenharia Química e \\ Estatística \\ ${ }^{2}$ Universidade Federal de Minas Gerais, Departamento de Engenharia Química \\ ${ }^{3}$ Universidade Federal de São João del-Rei, Departamento de Engenharias de
}

Telecomunicações e Mecatrônica, e Centro Federal de Educação Tecnológica de Minas

Gerais, Departamento de Engenharia Elétrica

E-mail para contato: galmeida@deq.ufmg.br

\begin{abstract}
RESUMO - Em qualquer parte do mundo, o papel da engenharia de processos é cada vez mais relevante para a própria manutenção dos negócios das indústrias químicas, dado um ambiente cada vez mais competitivo. Nesse trabalho, implementou-se um modelo do sistema de evaporação múltiplo efeito da indústria de celulose Kraft. Após validação, empregou-se o modelo para a simulação e análise de cenários operacionais usuais, quando é possível observar e comparar os efeitos sobre variáveis, intermediárias e de saída, a partir de perturbações, de magnitudes variadas, em diferentes variáveis de entrada. Ao final, observou-se a maior influência da temperatura do vapor vivo em relação à concentração inicial do licor sobre a demanda de vapor vivo, a temperatura do licor de saída do terceiro efeito, e a capacidade de transferência de calor no primeiro efeito.
\end{abstract}

\section{INTRODUÇÃO}

A simulação de cenários operacionais em processos químicos sempre foi uma etapa importante em análise de processos (Perlingeiro, 2005). Em função de sua crescente complexidade, maior integração entre unidades de processamento e outros fatores, a execução de perturbações in loco é uma atividade cada vez mais difícil, principalmente devido à questão de segurança operacional. Pelos mesmos fatores, com regulações mais restritas, seja da sociedade, de governos e de associações, a necessidade pela simulação de cenários operacionais é crescente. Alguns dos objetivos são, a verificação de robustez do processo, a identificação de gargalos nas operações, e a realização de testes de alterações em matériasprimas ou condições operacionais. Atualmente, a aquisição de informações até então desconhecidas, a favor de ganhos econômicos e produção mais limpa, é um fator essencial para a própria manutenção do negócio.

O presente trabalho utiliza um sistema de evaporação múltiplo efeito da indústria de celulose Kraft com o foco em simulação de cenários operacionais. O setor de celulose é responsável pela produção de papel, a partir do processo de separação das fibras de celulose da madeira, conhecida como polpação. O principal meio de obtenção de polpa celulósica em 
todo o mundo é o processo Kraft, que faz uso de uma solução rica em hidróxido de sódio e sulfeto de sódio, denominada licor de cozimento (Assumpção et al., 1988). O processo de obtenção de pasta celulósica é composto por duas unidades principais: a linha de fibras, cuja principal etapa é a polpação da madeira, e a unidade de recuperação química, responsável por tratar o licor negro, subproduto da polpação. Esse licor, com percentual mássico entre 15 e $20 \%$, é concentrado em um sistema de evaporação múltiplo efeito antes de sua combustão em uma caldeira para recuperação do licor de cozimento e geração de vapor (Vakkilainen, 1999).

A Figura 1 é um esquema de um sistema de evaporação múltiplo, composto por três efeitos (em série), sendo que seis é a quantidade usual. Um efeito é composto por um ou mais corpos de evaporação. A concentração do licor de saída é superior a $60 \%$ em massa, sendo que fábricas modernas operam com concentrações entre 80 e $85 \%$. A motivação em sistemas múltiplo efeito é a economia de energia, ao aproveitar o calor latente do vapor gerado em um efeito como meio de aquecimento para efeito subsequente. A pressão de operação é decrescente entre os efeitos, sendo maior no primeiro e negativa no último. Em um sistema de seis efeitos, a alimentação de vapor vivo é no primeiro efeito, e a de licor, em geral, a partir do quarto efeito. Desse modo, há um sistema misto entre as correntes de vapor e de licor, inicialmente co-corrente, e posteriormente, contracorrente. As principais métricas de operação de sistemas múltiplo efeito são, a capacidade de evaporação, dada em número de toneladas de água evaporada por hora, e a economia de vapor, dada pela razão entre número de toneladas de água evaporada por tonelada de vapor vivo (Venkatesh e Nguyen, 1992). A simulação de cenários operacionais em plantas de evaporação é importante em estudos de alterações de condições operacionais e de otimização, com o propósito de melhoria de desempenho. Um cenário extensivamente estudado atualmente é quanto a retirada de parte da matéria orgânica do licor (lignina) para outros fins e o efeito potencial no processo subsequente de combustão do licor na caldeira (não abordado nesse trabalho). Nesse trabalho, simularam-se perturbações positivas e negativas, com magnitudes variadas, em duas variáveis de entrada e observou-se os efeitos em três variáveis calculadas do primeiro efeito.

Figura 1 - Sistema de evaporação múltiplo efeito (Holmlund e Parviainen, 1999)

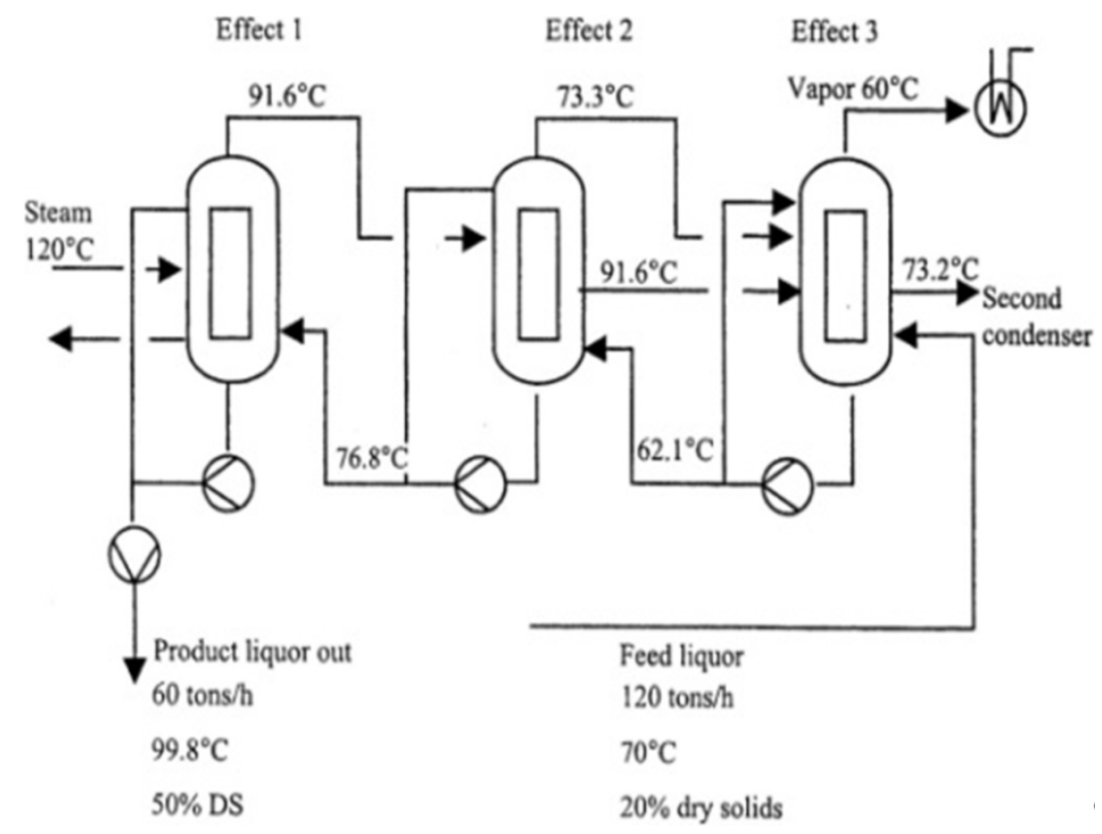




\section{OBJETIVO GERAL}

Implementar o modelo de Holmlund e Parviainen (1999), de um sistema de evaporação múltiplo efeito do processo Kraft, composto por três efeitos, e empregá-lo para a simulação de cenários operacionais.

\section{METODOLOGIA}

\subsection{Implementação do modelo de Holmlund e Parviainen (1999)}

Holmlund e Parviainen (1999) apresentaram um modelo simplificado de um sistema de evaporação múltiplo efeito para o licor negro do processo Kraft, composto por três corpos de evaporação (efeitos) (Figura 1). O licor, com entrada no terceiro efeito e saída no primeiro efeito, é concentrado de $20 \%$ para $50 \%$ em massa. As correlações empíricas para o calor específico do licor $\left(C_{p}\right.$, em $\mathrm{kJ} /(\mathrm{kg} \cdot \mathrm{K})$; entrada e efeitos 1 a 3) e a Elevação do Ponto de Ebulição (EPE, em K; efeitos 1, 2 e 3) são conforme as Equações 1 (Masse et al., 1986) e 2, em que X e T são, respectivamente, a concentração em massa (em \%) e a temperatura do licor $\left({ }^{\circ} \mathrm{C}\right)$, e $T_{P}$ é a temperatura de ebulição da água a pressão $\mathrm{p}(\mathrm{em} \mathrm{K})$. O EPE é a diferença entre a temperatura de ebulição da solução de licor e aquela da água pura à mesma pressão. Quanto maior a concentração do licor, maior a diferença. A entalpia do vapor e do condensado, ou seja, o calor latente, é calculado a partir da tabela de vapor. Para aproveitamento de calor, considera-se um tanque flash para o condensado de saída do segundo e terceiro efeitos. Entre outras considerações, estão a ausência de perda de carga entre os efeitos e a ausência de perda de energia com gases não condensáveis a partir dos efeitos. A Tabela 1 contém o conjunto de variáveis de entrada, com os respectivos valores. Validou-se o modelo com o cálculo do erro relativo percentual $\left(=\left(x-x^{*}\right) / x^{*} \cdot 100\right)$.

$$
\begin{aligned}
& C_{p}=4,216(1-X)+\left(1,675+\frac{3,31 T}{1000}\right) X+\left(4,87-\frac{20 T}{1000}\right)(1-X) X^{3} \\
& E P E=\left(6,173 X-7,48 X^{1,5}+32,747 X^{2}\right)\left(1+0,6\left(T_{p}-3,7316\right) / 100\right)
\end{aligned}
$$

Tabela 1 - Conjunto de variáveis de entrada

\begin{tabular}{|c|c|}
\hline Variável & Valor \\
\hline Concentração do licor (entrada) & 0,20 \\
\hline Concentração do licor (saída) & 0,50 \\
\hline Temperatura do licor (entrada-efeito $\left.3 ;{ }^{\circ} \mathrm{C}\right)$ & 70 \\
\hline Capacidade evaporação $(\mathrm{kg} / \mathrm{s})$ & 30 \\
\hline Temperatura (de saturação) do vapor vivo (entrada-efeito $\left.1 ;{ }^{\circ} \mathrm{C}\right)$ & 120 \\
\hline Temperatura (de saturação) do vapor (saída-efeito $\left.3 ;{ }^{\circ} \mathrm{C}\right)$ & 60 \\
\hline Vazão mássica de sólidos secos (saída-efeito $1 ; \mathrm{kg} / \mathrm{s})$ & 10 \\
\hline Coeficiente global de transferência de calor (efeito $1 ; \mathrm{kW} /\left(\mathrm{m}^{2} \cdot{ }^{\circ} \mathrm{C}\right)$ ) & 1,2 \\
\hline Coeficiente global de transferência de calor (efeito $2 ; \mathrm{kW} /\left(\mathrm{m}^{2} \cdot{ }^{\circ} \mathrm{C}\right)$ ) & 1,6 \\
\hline Coeficiente global de transferência de calor (efeito $3 ; \mathrm{kW} /\left(\mathrm{m}^{2} \cdot{ }^{\circ} \mathrm{C}\right)$ ) & 2,0 \\
\hline Área de transferência de calor (efeitos $\left.1,2 \mathrm{e} 3 ; \mathrm{m}^{2}\right)$ & 1040 \\
\hline
\end{tabular}




\subsection{Simulação de cenários operacionais}

Após implementação e validação do modelo de Holmlund e Parviainen (1999), simulou-se um conjunto de cenários operacionais, a partir de perturbações de $\pm 1,5,10$ e $15 \%$ (em relação ao ponto nominal de operação) em um conjunto de variáveis de entrada (Tabela 1).

\section{APRESENTAÇÃO E DISCUSSÃO DOS RESULTADOS}

A Tabela 2 mostra o resultado e a comparação para um conjunto de variáveis calculadas. Pode-se observar, por completo, a baixa magnitude do erro relativo percentual. Portanto, validou-se a implementação do modelo de Holmlund e Parviainen (1999).

Tabela 2 - Resultado e comparação entre modelos para variáveis calculadas

\begin{tabular}{|c|c|c|c|}
\hline Variável & $\begin{array}{c}\text { Presente } \\
\text { trabalho }\end{array}$ & $\begin{array}{c}\text { Holmlund e } \\
\text { Parviainen } \\
\text { (1999) }\end{array}$ & $\begin{array}{c}\text { Erro } \\
\text { relativo } \\
\text { (em \%) }\end{array}$ \\
\hline \hline Concentração do licor (saída-efeito 3) & 0,25 & 0,25 & 0,000 \\
\hline Elevação do ponto de ebulição (efeito 3; $\left.{ }^{\circ} \mathrm{C}\right)$ & 2,15 & 2,1 & 2,381 \\
\hline Temperatura do licor (saída-efeito $\left.1 ;{ }^{\circ} \mathrm{C}\right)$ & 100,85 & 99,8 & 1,052 \\
\hline Temperatura (de saturação) do vapor $\left(\right.$ saída-efeito $\left.1 ;{ }^{\circ} \mathrm{C}\right)$ & 92,62 & 91,6 & 1,114 \\
\hline Calor específico do licor (efeito $1 ; \mathrm{kJ} /\left(\mathrm{kg} \cdot{ }^{\circ} \mathrm{C}\right)$ ) & 3,04 & 3,0 & 1,333 \\
\hline Demanda de energia (efeito $1 ; \mathrm{kJ} / \mathrm{s})$ & 24820 & 24819 & 0,004 \\
\hline Produção (demanda) de vapor $($ efeito $1 ; \mathrm{kg} / \mathrm{s})$ & 11,30 & 11,3 & 0,000 \\
\hline Condensado (tanque flash do efeito 1 para o efeito2; $\mathrm{kg} / \mathrm{s})$ & 0,62 & 0,6 & 3,333 \\
\hline Capacidade de transferência de calor $(\mathrm{efeito} 1 ; \mathrm{kJ} / \mathrm{s})$ & 23899 & 25240 & $-5,313$ \\
\hline
\end{tabular}

Em seguida, utilizou-se o modelo para a simulação de cenários, com perturbações em variáveis de entrada (seção 3.2). A Tabela 3 é um quadro-resumo com os resultados para perturbações (em relação ao ponto nominal; Tabela 1) positivas $(+1 \%,+5 \%$ e $+10 \%)$ e negativas $(-1 \%,-5 \%$ e $-10 \%)$ na concentração do licor de entrada e na temperatura (de saturação) do vapor vivo. Analisaram-se os efeitos sobre um conjunto de variáveis de saída do primeiro efeito. $\mathrm{O}$ estudo da demanda de vapor é importante pela relação direta com o consumo de energia; já a análise da temperatura do licor de saída é importante por questões de eficiência operacional e de bombeamento, por causa da viscosidade do licor; e a investigação sobre a capacidade de transferência de calor também é importante pela relação direta com a eficiência operacional. A Figura 2 mostra os resultados através de gráficos. Pode-se observar, em geral, a maior influência da temperatura do vapor vivo em relação à concentração do licor de entrada, cujo maior efeito é sobre a demanda de vapor. Ainda, as mudanças significativas sobre a capacidade de transferência de calor. 
Tabela 3 - Resultado das perturbações, positivas e negativas, na concentração do licor de entrada, com ponto nominal de 0,20 (Tabela 1)

\begin{tabular}{|c|c|c|c|c|c|}
\hline \multirow[t]{3}{*}{ Variável } & \multirow[t]{3}{*}{\begin{tabular}{c|} 
Ponto \\
nominal
\end{tabular}} & \multicolumn{4}{|c|}{ Perturbação } \\
\hline & & \multicolumn{4}{|c|}{$\begin{array}{c}\text { Concentração do licor de entrada } \\
\text { (ponto nominal: } \mathbf{0 , 2 0} \text { ) }\end{array}$} \\
\hline & & $+1 \%$ & $+5 \%$ & $+10 \%$ & $+15 \%$ \\
\hline Produção (demanda) de vapor (efeito $1 ; \mathrm{kg} / \mathrm{s}$ ) & 11,3 & 11,31 & 11,37 & 11,43 & 11,50 \\
\hline Temperatura do licor (saída-efeito $1 ;{ }^{\circ} \mathrm{C}$ ) & 99,8 & 100,88 & 101,01 & 101,16 & 101,32 \\
\hline \multirow[t]{2}{*}{ Capacidade de transferência de calor (efeito $1 ; \mathrm{kJ} / \mathrm{s}$ ) } & 23899 & 23860 & 23704 & 23510 & 23316 \\
\hline & & $-1 \%$ & $-5 \%$ & $-10 \%$ & $-15 \%$ \\
\hline Produção (demanda) de vapor (efeito $1 ; \mathrm{kg} / \mathrm{s}$ ) & 11,3 & 11,29 & 11,23 & 11,16 & 11,09 \\
\hline Temperatura do licor (saída-efeito $1 ;{ }^{\circ} \mathrm{C}$ ) & 99,8 & 100,82 & 100,69 & 100,54 & 100,38 \\
\hline \multirow[t]{3}{*}{ Capacidade de transferência de calor (efeito $1 ; \mathrm{kJ} / \mathrm{s}$ ) } & 23899 & 23938 & 24095 & 24291 & 24487 \\
\hline & & \multicolumn{4}{|c|}{$\begin{array}{c}\text { Temperatura (de saturação) do } \\
\text { vapor vivo (ponto nominal: } 120^{\circ} \mathrm{C} \text { ) }\end{array}$} \\
\hline & & $+1 \%$ & $+5 \%$ & $+10 \%$ & $+15 \%$ \\
\hline Produção (demanda) de vapor (efeito $1 ; \mathrm{kg} / \mathrm{s}$ ) & 11,3 & 11,33 & 11,44 & 11,59 & 11,74 \\
\hline Temperatura do licor (saída-efeito $1 ;{ }^{\circ} \mathrm{C}$ ) & 99,8 & 101,55 & 104,35 & 107,85 & 111,35 \\
\hline \multirow[t]{2}{*}{ Capacidade de transferência de calor (efeito $1 ; \mathrm{kJ} / \mathrm{s}$ ) } & 23899 & 24593 & 27019 & 30139 & 33259 \\
\hline & & $-1 \%$ & $-5 \%$ & $-10 \%$ & $-15 \%$ \\
\hline Produção (demanda) de vapor (efeito $1 ; \mathrm{kg} / \mathrm{s}$ ) & 11,3 & 11,27 & 11,16 & 11,02 & 10,89 \\
\hline Temperatura do licor (saída-efeito $1 ;{ }^{\circ} \mathrm{C}$ ) & 99,8 & 100,15 & 97,35 & 93,85 & 90,35 \\
\hline Capacidade de transferência de calor (efeito $1 ; \mathrm{kJ} / \mathrm{s}$ ) & 23899 & 23275 & 20779 & 17659 & 14539 \\
\hline
\end{tabular}

Figura 2 - Resultado das perturbações na concentração do licor de entrada e na temperatura (de saturação) do vapor vivo (pontos nominais: 0,20 e $120^{\circ} \mathrm{C}$, respectivamente; Tabela 1 )

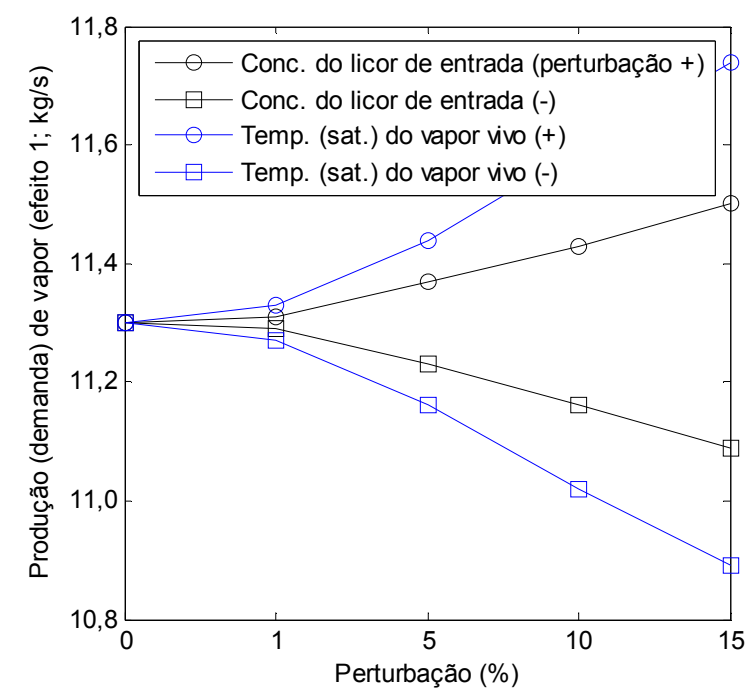

(a) Efeito sobre a produção (demanda) de vapor no primeiro efeito

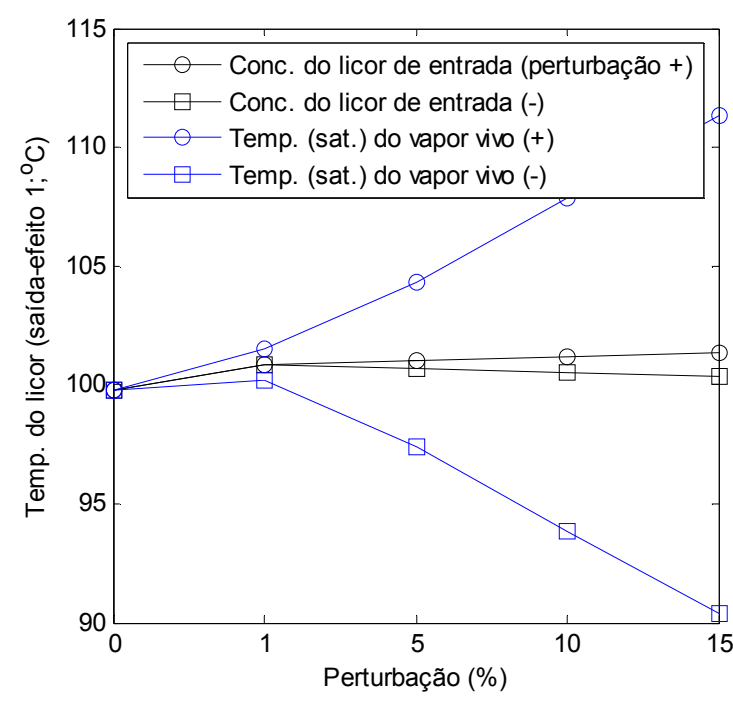

(b) Efeito sobre a temperatura do licor de saída no primeiro efeito 
Figura 2 (continuação)

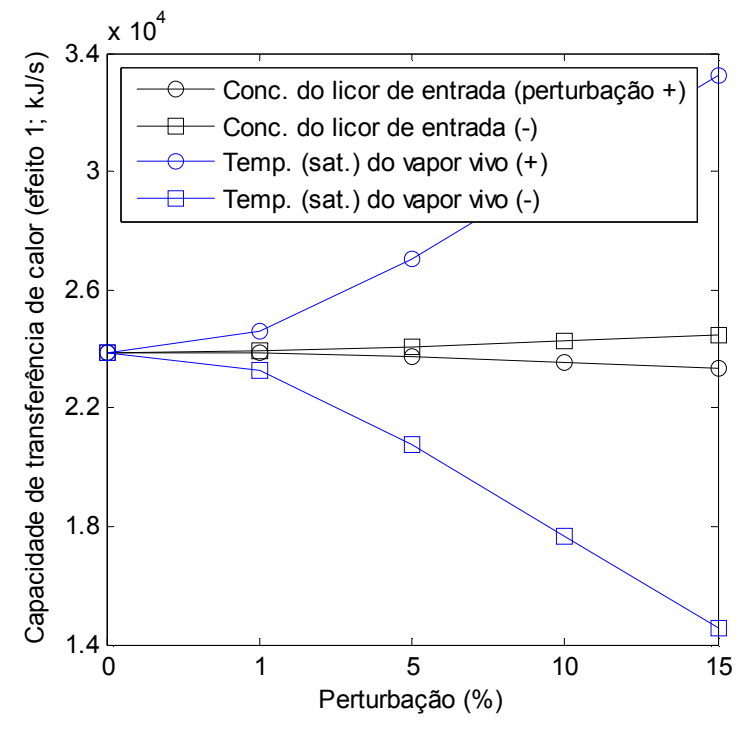

(c) Efeito sobre a capacidade de transferência de calor no primeiro efeito

\section{CONSIDERAÇÕES FINAIS}

Empregou o modelo de Holmlund e Parviainen (1999), de um sistema de evaporação múltiplo efeito, para um estudo de simulação de cenários operacionais. Após a sua implementação e validação, simularam-se perturbações positivas e negativas, com magnitudes variadas, em duas variáveis de entrada e observaram-se os efeitos em três variáveis calculadas do primeiro efeito. Em geral, esse tipo de análise é importante para verificar o comportamento do processo em torno do ponto nominal de operação; entender melhor os relacionamentos entre variáveis de entrada, intermediárias e de saída; identificar gargalos e pontos de melhoria; e construir modelos mais complexos.

\section{BIBLIOGRAFIA}

ASSUMPÇÃO, R. M. V.; CAHEN, R.; PHILIPP, P. Celulose e Papel: Tecnologia de Fabricação da Pasta Celulósica, v. 1, 2a ed., São Paulo: IPT e SENAI, 1988. Cap. VI, p. 169-319.

HOLMLUND, K.; PARVIAINEN, K. Evaporation of Black Liquor. In: Gullichsen, J. e Fogelholm, C-J. (eds.), Chemical pulping (Book 6B). Atlanta: TAPPI Press, 1999, p. 396-417.

MASSE, M.A.; KIRAN, E.; FRICKE, A.L. Freezing and Glass Transition Phenomena in Polymer-Diluent Mixtures, Polymer, v. 27, p. 619-622, 1986.

PERlingeIRO, C. A. G. Engenharia de Processos: Análise, Simulação, Otimização e Sintese de Processos Químicos, Brasil: Blucher, 2005.

VAKKILAINEN, E. Chemical Recovery. In: Gullichsen, J. e Fogelholm, C.-J. (eds.), Chemical pulping (Book 6B). Atlanta: TAPPI Press, 1999, p. 396-417.

VENKATESH, V.; NGUYEN, X. N., Evaporation and Concentration of Black Liquor. In: Green, R. P., Hough, G. (eds), Chemical Recovery in The Alkaline Pulping Processes, 3a ed. Atlanta: TAPPI Press, 1992. 